\title{
ON INITIAL BOUNDARY VALUE PROBLEM WITH DIRICHLET INTEGRAL CONDITIONS FOR A HYPERBOLIC EQUATION WITH THE BESSEL OPERATOR
}

\section{ABDELFATAH BOUZIANI}

Received 12 April 2002 and in revised form 15 June 2003

We consider a mixed problem with Dirichlet and integral conditions for a second-order hyperbolic equation with the Bessel operator. The existence, uniqueness, and continuous dependence of a strongly generalized solution are proved. The proof is based on an a priori estimate established in weighted Sobolev spaces and on the density of the range of the operator corresponding to the abstract formulation of the considered problem.

\section{Introduction}

In the recent years, hyperbolic equations with integral condition(s) have received considerable attention. The physical significance of these conditions (mean, total mass, moments, etc.) has served as a fundamental reason for the increasing interest carried to this type of problems. For instance, many processes in porous media can be described by secondorder hyperbolic equations with an integral condition $[14,15]$. The presence of an integral term in boundary conditions can greatly complicate the application of standard functional or numerical methods, owing to the fact that the elliptic differential operator with integral condition is no longer positive definite in the usual function spaces, which poses the main source of difficulty. What returns the adaptation of these methods to this type of problems is a subject of topicality. Therefore, the investigation of these problems requires a separate study at every time.

In this paper, we are concerned with a boundary value problem with an integral condition for a second-order hyperbolic equation with the Bessel operator. It can be a part in the contribution of the development of the energy-integral method for solving such problems. 
The precise statement of the problem is as follows: let $b, T>0, \Omega=$ $(0, b), I=(0, T)$, and $Q=\left\{(x, t) \in \mathbb{R}^{2}: x \in \Omega, t \in I\right\}$. Find a function $\theta=$ $\theta(x, t)$ satisfying the equation

$$
\left\llcorner\theta=\frac{\partial^{2} \theta}{\partial t^{2}}-\frac{1}{x} \frac{\partial}{\partial x}\left(x \frac{\partial \theta}{\partial x}\right)=h(x, t),\right.
$$

the initial conditions

$$
\begin{aligned}
& \ell_{0} \theta=\theta(x, 0)=\theta_{0}(x), \\
& \ell_{1} \theta=\frac{\partial \theta(x, 0)}{\partial t}=\theta_{1}(x),
\end{aligned}
$$

the Dirichlet condition

$$
\theta(b, t)=\mu(t),
$$

and the integral condition

$$
\frac{1}{b} \int_{\Omega} \theta(x, t) d x=m(t) .
$$

If $\theta=\theta(x, t)$ is the ground-water level at point $x \in \Omega$ at time $t$, then $m(t)$ is the mean value of $\theta(x, t)$ at time $t$.

The regular case of this problem has been treated in [1] by using Fourier's method to prove the existence and uniqueness of a classical solution. Similar equation with Neumann weighted integral conditions has been studied in [12]. Mixed problems for second-order hyperbolic equations, when in (1.1a) instead of the Bessel operator we have the operator $(\partial / \partial x)(a(x, t)(\partial u / \partial x))$ with Neumann integral conditions, are investigated in $[3,4,7,8]$. As for hyperbolic equations with only integral conditions, they have been studied in $[3,7,13,15,16]$. Concerning problems with integral conditions for other equations, we refer the reader, for instance, to $[2,5,6]$ and the references therein.

In this paper, following the method presented, for instance, in [6], we prove that problem (1.1) possesses a unique strongly generalized solution, in weighted Sobolev spaces, that depends continuously on the right-hand side of (1.1a), the initial conditions (1.1b) and (1.1c), and the boundary conditions (1.1d) and (1.1e).

\section{Notation, assumptions, and some auxiliary inequalities}

First, we assume the following:

(A1) $\mu, m \in C^{2}(I)$;

(A2) the compatibility conditions 


$$
\begin{array}{ll}
\theta_{0}(b)=\mu(0), & \frac{1}{b} \int_{\Omega} \theta_{0}(x) d x=m(0), \\
\theta_{1}(b)=\mu^{\prime}(0), & \frac{1}{b} \int_{\Omega} \theta_{1}(x) d x=m^{\prime}(0) .
\end{array}
$$

Then, we reduce problem (1.1) to an equivalent problem with homogeneous boundary conditions by introducing a new unknown function $u$ defined by $u(x, t)=\theta(x, t)-U(x, t)$ with

$$
U(x, t)=(2 m(t)-\mu(t))+\frac{2 x}{b}(\mu(t)-m(t)) .
$$

Therefore, problem (1.1) can be formulated as follows. Find a function $u=u(x, t)$ satisfying

$$
\begin{gathered}
\mathcal{L}_{u}=\frac{\partial^{2} u}{\partial t^{2}}-\frac{1}{x} \frac{\partial}{\partial x}\left(x \frac{\partial u}{\partial x}\right)=f(x, t), \\
\ell_{0} u=u(x, 0)=u_{0}(x), \\
\ell_{1} u=\frac{\partial u(x, 0)}{\partial t}=u_{1}(x), \\
u(b, t)=0, \\
\int_{\Omega} u(x, t) d x=0,
\end{gathered}
$$

with the compatibility conditions

$$
u_{i}(b)=0, \quad \int_{\Omega} u_{i}(x) d x=0, \quad(i=0,1)
$$

where $f(x, t)=h(x, t)-\mathcal{L} U, u_{0}(x)=\theta_{0}(x)-\ell_{0} U$, and $u_{1}(x)=\theta_{1}(x)-\ell_{1} U$.

We now introduce appropriate function spaces. Let $L^{2}(\Omega)$ be the usual space of square integrable functions and let $L_{\rho}^{2}(\Omega)$ be the weighted $L^{2}$ space with finite norm

$$
\|u\|_{L_{\rho}^{2}(\Omega)}^{2}=\int_{\Omega} \rho u^{2} d x
$$

and with associated inner product

$$
(u, v)_{L_{\rho}^{2}(\Omega)}=\int_{\Omega} \rho u v d x .
$$


We denote by $V_{\sigma}^{1}(\Omega)$ the Hilbert space obtained by endowing $C^{1}(\Omega)$ with the norm

$$
\|u\|_{V_{\sigma}^{1}(\Omega)}^{2}=\int_{\Omega}\left(x^{2} u^{2}+x\left(\frac{\partial u}{\partial x}\right)^{2}\right) d x
$$

and the associated inner product

$$
(u, v)_{V_{\sigma}^{1}(\Omega)}=\int_{\Omega} x^{2} u v d x+\int_{\Omega} x \frac{\partial u}{\partial x} \frac{\partial v}{\partial x} d x .
$$

Let $B_{2}^{1}(I)$ be the space first introduced by the author in $[2,3,4,5]$ as the completion of the space $C_{0}(I)$ of real continuous functions with compact support in $I$ with respect to the inner product

$$
(u, v)_{B_{2}^{m}(I)}=\int_{I} \Im_{t}^{m} u \Im_{t}^{m} v d t
$$

where

$$
\Im_{t}^{m} u=\int_{0}^{t} \frac{(t-\tau)^{m-1}}{(m-1) !} u(x, \tau) d \tau,
$$

for every fixed $t \in I$. The corresponding norm is

$$
\|u\|_{B_{2}^{m}(I)}=\sqrt{(u, u)_{B_{2}^{m}(I)}} .
$$

We also use the standard function spaces $C(\bar{I}, H)$ and $L^{2}(I, H)$ of continuous and $L^{2}$-Bochner integrable mappings from $\bar{I}$ onto a Banach space $H$, respectively (see [9]). Let $B_{2}^{m}(I, H)$ be the space of functions from $I$ into $H$ which is a Bouziani space for the measure $d t$. It is a Hilbert space for the finite norm

$$
\|u\|_{B_{2}^{m}(I, H)}=\left(\int_{I}\left(\left\|\Im_{t}^{m} u\right\|_{H}\right)^{2} d t\right)^{1 / 2} .
$$

This is the case, for instance, when $H=L^{2}(\Omega), H=L_{\rho}^{2}(\Omega)$ (with $\rho=x$ or $\left.\rho=x^{2}\right)$, or $H=V_{\sigma}^{1}(\Omega)$.

Problem (2.3) can be viewed as the problem of solving the operator equation

$$
L u=\mathscr{F}
$$


where $\mathcal{F}=\left(f, u_{0}, u_{1}\right)$ and $L$ is the operator given by

$$
L u=\left(\left\llcorner u, \ell_{0} u, \ell_{1} u\right)\right.
$$

We consider $L$ as an unbounded operator with the domain $D(L)$ consisting of all functions $u$ belonging to $L^{2}\left(I, L_{x}^{2}(\Omega)\right)$ for which $\partial^{p} u / \partial t^{p}$, $\partial^{p} u / \partial x^{p}(p=1,2), \partial^{2} u / \partial t \partial x \in L^{2}\left(I, L_{x}^{2}(\Omega)\right)$ and satisfying conditions (2.3d) and (2.3e). Let $B$ be the Banach space obtained by the closure of $D(L)$ in the norm

$$
\|u\|_{B}=\left(\|u\|_{C\left(\bar{I}, V_{\sigma}^{1}(\Omega)\right)}^{2}+\left\|\frac{\partial u}{\partial t}\right\|_{C\left(\bar{I}, L_{x}^{2}(\Omega)\right)}^{2}\right)^{1 / 2}
$$

while $F$ is the Hilbert space $L^{2}\left(I, L_{x}^{2}(\Omega)\right) \times V_{\sigma}^{1}(\Omega) \times L_{x}^{2}(\Omega)$ consisting of vector-valued functions $\mathcal{F}=\left(f, u_{0}, u_{1}\right)$ for which the norm

$$
\|\mathcal{F}\|_{F}=\left(\|f\|_{L^{2}\left(I, L_{x}^{2}(\Omega)\right)}^{2}+\left\|u_{0}\right\|_{V_{\sigma}^{1}(\Omega)}^{2}+\left\|u_{1}\right\|_{L_{x}^{2}(\Omega)}^{2}\right)^{1 / 2}
$$

is finite. The associated inner product is

$$
(\mathcal{F}, W)_{F}=(f, \omega)_{L^{2}\left(I, L_{x}^{2}(\Omega)\right)}+\left(u_{0}, \omega_{0}\right)_{V_{\sigma}^{1}(\Omega)}+\left(u_{1}, \omega_{1}\right)_{L_{x}^{2}(\Omega)} .
$$

The elements $u$ are continuous functions on $\bar{I}$ with values in $V_{\sigma}^{1}(\Omega)$ and have derivatives $\partial u / \partial t$ which are continuous on $\bar{I}$ with values in $L_{x}^{2}(\Omega)$. Hence, the mappings

$$
\begin{aligned}
& \ell_{0}: B \ni u \longrightarrow \ell_{0} u=\left.u\right|_{t=0} \in V_{\sigma}^{1}(\Omega), \\
& \ell_{1}: B \ni u \longrightarrow \ell_{1} u=\left.\frac{\partial u}{\partial t}\right|_{t=0} \in L_{x}^{2}(\Omega)
\end{aligned}
$$

are defined and continuous on $B$.

Throughout the paper, we use the following operators:

$$
\begin{array}{ll}
\Im_{t} u=\int_{0}^{t} u(\cdot, s) d s, & \Im_{t}^{*} u=\int_{t}^{T} u(\cdot, s) d s, \\
\Im_{t-\tau}^{*} u=\Im_{t}^{*} u-\Im_{\tau}^{*} u, & \Im_{x}^{*} u=\int_{x}^{b} u(\xi, \cdot) d \xi .
\end{array}
$$


It is easy to check that the following result holds:

$$
\begin{gathered}
-\frac{\partial}{\partial t} \supset_{t-\tau}^{*} u=-\frac{\partial}{\partial t} \Im_{t}^{*} u=\frac{\partial}{\partial t} \Im_{t} u=-\frac{\partial}{\partial x} \Im_{x}^{*} u=u, \\
\Im_{0}^{*} u=0, \quad \supset_{-\tau}^{*} u=\Im_{\tau} u, \quad \Im_{0} u=0, \quad \Im_{b}^{*} u=0,
\end{gathered}
$$

for all $t, \tau \in[0, T]$ and $x \in[0, b]$.

However, the following known inequalities are frequently used. We list them here for convenience.

(1) The Schwarz inequality. For $g, h \in L^{2}(0, T)$, we have

$$
\left(\int_{I} g(t) h(t) d t\right)^{2} \leq\left(\int_{I} g^{2}(t) d t\right) \cdot\left(\int_{I} h^{2}(t) d t\right) .
$$

(2) The Cauchy inequality. For any real $a, b$, and $\varepsilon>0$, we have

$$
a b \leq \frac{\varepsilon}{2} a^{2}+\frac{1}{2 \varepsilon} b^{2} .
$$

(3) The Gronwall lemma [10, page 56] and [11, Lemma 7.1]. If $f_{i}$ (where $i=1,2)$ are nonnegative functions on $I, f_{1}$ is integrable on $I$, and $f_{2}$ is bounded nondecreasing in $I$, and $c$ is a positive constant, then

$$
f_{1}(\tau) \leq e^{c \tau} f_{2}(\tau)
$$

is a direct consequence of the inequality

$$
f_{1}(\tau) \leq c \int_{0}^{\tau} f_{1}(s) d s+f_{2}(\tau)
$$

(4) Moreover, we have

$$
\begin{gathered}
\int_{0}^{T}\left(\Im_{t}^{*} u\right)^{2} d t \leq \frac{T^{2}}{2} \int_{0}^{T} u^{2} d t \\
\int_{0}^{T}\left(\supset_{t-\tau}^{*} u\right)^{2} d t \leq 2 T^{2} \int_{0}^{T} u^{2} d t \\
\int_{\Omega}\left(\Im_{x}^{*} u\right)^{2} d x \leq 4 \int_{\Omega} x^{2} u^{2} d x .
\end{gathered}
$$

\section{Uniqueness and continuous dependence}

First we establish an a priori estimate from which we conclude the uniqueness and continuous dependence of the solution with respect to the right-hand side of (2.3a) and on the initial conditions (2.3b) and (2.3c). 
THEOREM 3.1. For any function $u$ belonging to $D(L)$, the following estimate holds:

$$
\|u\|_{B} \leq c\|L u\|_{F}
$$

where

$$
c=\sqrt{1+2 b} \exp \left(\frac{\max \left(10 T^{2}+5 T+4,1+b\right) T}{2}\right) .
$$

Proof. Taking the scalar product in $L^{2}(\Omega \times(0, \tau))$, with $0 \leq \tau \leq T$, of $(2.3 a)$ and the integrodifferential operator

$$
M u=x^{2} \supset_{t-\tau}^{*} u+x \supset_{t-\tau}^{*}\left(\Im_{x}^{*} u\right)+x \frac{\partial u}{\partial t}
$$

we have

$$
\begin{aligned}
\int_{0}^{\tau} \int_{\Omega} & \mathcal{L} u M u d x d t \\
= & \int_{0}^{\tau} \int_{\Omega} x^{2} \frac{\partial^{2} u}{\partial t^{2}} \supset_{t-\tau}^{*} u d x d t+\int_{0}^{\tau} \int_{\Omega} x \frac{\partial^{2} u}{\partial t^{2}} \supset_{t-\tau}^{*}\left(\Im_{x}^{*} u\right) d x d t \\
& +\int_{0}^{\tau} \int_{\Omega} x \frac{\partial^{2} u}{\partial t^{2}} \frac{\partial u}{\partial t} d x d t-\int_{0}^{\tau} \int_{\Omega} x \frac{\partial}{\partial x}\left(x \frac{\partial u}{\partial x}\right) \supset_{t-\tau}^{*} u d x d t \\
& -\int_{0}^{\tau} \int_{\Omega} \frac{\partial}{\partial x}\left(x \frac{\partial u}{\partial x}\right) \supset_{t-\tau}^{*}\left(\Im_{x}^{*} u\right) d x d t-\int_{0}^{\tau} \int_{\Omega} \frac{\partial}{\partial x}\left(x \frac{\partial u}{\partial x}\right) \frac{\partial u}{\partial t} d x d t
\end{aligned}
$$

Integrating by parts each term of equality (3.4), we obtain

$$
\begin{aligned}
& \int_{0}^{\tau} \int_{\Omega} x^{2} \frac{\partial^{2} u}{\partial t^{2}} J_{t-\tau}^{*} u d x d t \\
& =\left.\int_{\Omega} x^{2} \frac{\partial u}{\partial t} \supset_{t-\tau}^{*} u\right|_{0} ^{\tau} d x+\int_{0}^{\tau} \int_{\Omega} x^{2} \frac{\partial u}{\partial t} u d x d t \\
& =-\int_{\Omega} x^{2} u_{1} \Im_{\tau} u d x+\frac{1}{2} \int_{\Omega} x^{2} u^{2}(x, \tau) d x-\frac{1}{2} \int_{\Omega} x^{2} u_{0}^{2}(x) d x \\
& \int_{0}^{\tau} \int_{\Omega} x \frac{\partial^{2} u}{\partial t^{2}} \supset_{t-\tau}^{*}\left(\Im_{x}^{*} u\right) d x d t \\
& =\left.\int_{\Omega} x \frac{\partial u}{\partial t} \supset_{t-\tau}^{*}\left(\Im_{x}^{*} u\right)\right|_{0} ^{\tau} d x+\int_{0}^{\tau} \int_{\Omega} x \frac{\partial u}{\partial t} \Im_{x}^{*} u d x d t \\
& =-\int_{\Omega} x u_{1} \Im_{\tau}\left(\Im_{x}^{*} u\right) d x+\int_{0}^{\tau} \int_{\Omega} x \frac{\partial u}{\partial t} \Im_{x}^{*} u d x d t
\end{aligned}
$$


494 On hyperbolic equation with an integral condition

$$
\begin{aligned}
& \int_{0}^{\tau} \int_{\Omega} x \frac{\partial^{2} u}{\partial t^{2}} \frac{\partial u}{\partial t} d x d t \\
& =\frac{1}{2} \int_{\Omega} x\left(\frac{\partial u(x, \tau)}{\partial t}\right)^{2} d x-\frac{1}{2} \int_{\Omega} x u_{1}^{2}(x) d x, \\
& -\int_{0}^{\tau} \int_{\Omega} x \frac{\partial}{\partial x}\left(x \frac{\partial u}{\partial x}\right) \supset_{t-\tau}^{*} u d x d t \\
& =-\left.\int_{0}^{\tau} x^{2} \frac{\partial u}{\partial x} \supset_{t-\tau}^{*} u\right|_{0} ^{b} d t+\int_{0}^{\tau} \int_{\Omega} x^{2} \frac{\partial u}{\partial x} \supset_{t-\tau}^{*}\left(\frac{\partial u}{\partial x}\right) d x d t \\
& +\int_{0}^{\tau} \int_{\Omega} x \frac{\partial u}{\partial x} \supset_{t-\tau}^{*} u d x d t \\
& =-\left.\frac{1}{2} \int_{\Omega} x^{2}\left(\supset_{t-\tau}^{*}\left(\frac{\partial u}{\partial x}\right)\right)^{2}\right|_{0} ^{\tau} d x+\int_{0}^{\tau} \int_{\Omega} x \frac{\partial u}{\partial x} \supset_{t-\tau}^{*} u d x d t \\
& =\frac{1}{2} \int_{\Omega} x^{2}\left(\Im_{\tau}\left(\frac{\partial u}{\partial x}\right)\right)^{2} d x+\int_{0}^{\tau} \int_{\Omega} x \frac{\partial u}{\partial x} \vartheta_{t-\tau}^{*} u d x d t, \\
& -\int_{0}^{\tau} \int_{\Omega} \frac{\partial}{\partial x}\left(x \frac{\partial u}{\partial x}\right) \mathcal{\supset}_{t-\tau}^{*}\left(\Im_{x}^{*} u\right) d x d t \\
& =-\left.\int_{0}^{\tau} x \frac{\partial u}{\partial x} \supset_{t-\tau}^{*}\left(\Im_{x}^{*} u\right)\right|_{0} ^{b} d t-\int_{0}^{\tau} \int_{\Omega} x \frac{\partial u}{\partial x} \jmath_{t-\tau}^{*} u d x d t \\
& =-\int_{0}^{\tau} \int_{\Omega} x \frac{\partial u}{\partial x} \Im_{t-\tau}^{*} u d x d t \\
& -\int_{0}^{\tau} \int_{\Omega} \frac{\partial}{\partial x}\left(x \frac{\partial u}{\partial x}\right) \frac{\partial u}{\partial t} d x d t \\
& =-\left.\int_{0}^{\tau} x \frac{\partial u}{\partial x} \frac{\partial u}{\partial t}\right|_{0} ^{b} d t+\int_{0}^{\tau} \int_{\Omega} x \frac{\partial u}{\partial x} \frac{\partial^{2} u}{\partial t \partial x} d x d t \\
& =\frac{1}{2} \int_{\Omega} x\left(\frac{\partial u(x, \tau)}{\partial x}\right)^{2} d x-\frac{1}{2} \int_{\Omega} x\left(u_{0}^{\prime}(x)\right)^{2} d x \text {. }
\end{aligned}
$$

Substituting (3.5) into (3.4), we obtain

$$
\begin{aligned}
\int_{\Omega} x^{2}\left(\Im_{\tau} \frac{\partial u}{\partial x}\right)^{2} d x+\int_{\Omega}\left(x^{2} u^{2}(x, \tau)+x\left(\frac{\partial u(x, \tau)}{\partial x}\right)^{2}+x\left(\frac{\partial u(x, \tau)}{\partial t}\right)^{2}\right) d x \\
=2 \int_{0}^{\tau} \int_{\Omega} \rho u M u d x d t+\int_{\Omega}\left(x^{2} u_{0}^{2}(x)+x\left(u_{0}^{\prime}(x)\right)^{2}+x u_{1}^{2}(x)\right) d x \\
\quad+2 \int_{\Omega} x^{2} u_{1} \Im_{\tau} u d x+2 \int_{\Omega} x u_{1} \Im_{\tau}\left(\Im_{x}^{*} u\right) d x-2 \int_{0}^{\tau} \int_{\Omega} x \frac{\partial u}{\partial t} \Im_{x}^{*} u d x d t .
\end{aligned}
$$


By virtue of the Cauchy inequality and inequalities (2.25), the first and the last terms on the right-hand side of (3.6) are estimated as follows:

$$
\begin{aligned}
& 2 \int_{0}^{\tau} \int_{\Omega} \mathcal{L} u M u d x d t \\
& \quad \leq(1+2 b) \int_{0}^{\tau} \int_{\Omega} x f^{2} d x d t \\
& \quad+10 T^{2} \int_{0}^{\tau} \int_{\Omega} x^{2} u^{2} d x d t+\int_{0}^{\tau} \int_{\Omega} x\left(\frac{\partial u}{\partial t}\right)^{2} d x d t \\
& -2 \int_{0}^{\tau} \int_{\Omega} x \frac{\partial u}{\partial t} \Im_{x}^{*} u d x d t \\
& \quad \leq b \int_{0}^{\tau} \int_{\Omega} x\left(\frac{\partial u}{\partial t}\right)^{2} d x d t+4 \int_{0}^{\tau} \int_{\Omega} x^{2} u^{2} d x d t .
\end{aligned}
$$

As for the third and fourth integrals on the right-hand side of (3.6), we use on top of that the Schwarz inequality to get

$$
\begin{aligned}
2 \int_{\Omega} x^{2} u_{1} \Im_{\tau} u d x & \leq \int_{\Omega} x^{2} u_{1}^{2} d x+\int_{\Omega} x^{2}\left(\Im_{\tau} u\right)^{2} d x \\
& \leq b \int_{\Omega} x u_{1}^{2} d x+T \int_{0}^{\tau} \int_{\Omega} x^{2} u^{2} d x d t \\
2 \int_{\Omega} x u_{1} \Im_{\tau}\left(\Im_{x}^{*} u\right) d x & \leq \int_{\Omega} x^{2} u_{1}^{2} d x+\int_{\Omega}\left(\Im_{\tau}\left(\Im_{x}^{*} u\right)\right)^{2} d x \\
& \leq b \int_{\Omega} x u_{1}^{2} d x+T \int_{0}^{\tau} \int_{\Omega}\left(\Im_{x}^{*} u\right)^{2} d x d t \\
& \leq b \int_{\Omega} x u_{1}^{2} d x+4 T \int_{0}^{\tau} \int_{\Omega} x^{2} u^{2} d x d t
\end{aligned}
$$

Inserting (3.7) and (3.8) into (3.6) and omitting the first term on the lefthand side of the obtained inequality, we get

$$
\begin{aligned}
& \int_{\Omega}(\left.x^{2} u^{2}(x, \tau)+x\left(\frac{\partial u(x, \tau)}{\partial x}\right)^{2}+x\left(\frac{\partial u(x, \tau)}{\partial t}\right)^{2}\right) d x \\
& \leq(1+2 b) \int_{0}^{\tau} \int_{\Omega} x f^{2} d x d t+\int_{\Omega}\left(x^{2} u_{0}^{2}(x)+x\left(u_{0}^{\prime}(x)\right)^{2}+x u_{1}^{2}(x)\right) d x \\
& \quad+2 b \int_{\Omega} x u_{1}^{2}(x) d x+\left(10 T^{2}+5 T+4\right) \int_{0}^{\tau} \int_{\Omega} x^{2} u^{2} d x d t \\
& \quad+(1+b) \int_{0}^{\tau} \int_{\Omega} x\left(\frac{\partial u}{\partial t}\right)^{2} d x d t
\end{aligned}
$$


496 On hyperbolic equation with an integral condition

from which we have

$$
\begin{aligned}
\| u(\cdot, \tau) & \left\|_{V_{\sigma}^{1}(\Omega)}^{2}+\right\| \frac{\partial u(\cdot, \tau)}{\partial t} \|_{L_{x}^{2}(\Omega)}^{2} \\
\leq & (1+2 b)\left(\int_{0}^{\tau}\|f(\cdot, t)\|_{L_{x}^{2}(\Omega)}^{2} d t+\left\|u_{0}\right\|_{V_{\sigma}^{1}(\Omega)}^{2}+\left\|u_{1}\right\|_{L_{x}^{2}(\Omega)}^{2}\right) \\
& +\max \left(10 T^{2}+5 T+4,1+b\right) \\
& \times \int_{0}^{\tau}\left(\|u(\cdot, t)\|_{V_{\sigma}^{1}(\Omega)}^{2}+\left\|\frac{\partial u(\cdot, t)}{\partial t}\right\|_{L_{x}^{2}(\Omega)}^{2}\right) d t .
\end{aligned}
$$

According to Gronwall's lemma, by putting

$$
\begin{aligned}
& f_{1}(\tau)=\|u(\cdot, \tau)\|_{V_{\sigma}^{1}(\Omega)}^{2}+\left\|\frac{\partial u(\cdot, \tau)}{\partial t}\right\|_{L_{x}^{2}(\Omega)}^{2}, \\
& f_{2}(\tau)=\int_{0}^{\tau}\|f(\cdot, t)\|_{L_{x}^{2}(\Omega)}^{2} d t+\left\|u_{0}\right\|_{V_{\sigma}^{1}(\Omega)}^{2}+\left\|u_{1}\right\|_{L_{x}^{2}(\Omega) \prime}^{2}
\end{aligned}
$$

we obtain

$$
\begin{aligned}
& \|u(\cdot, \tau)\|_{V_{\sigma}^{1}(\Omega)}^{2}+\left\|\frac{\partial u(\cdot, \tau)}{\partial t}\right\|_{L_{x}^{2}(\Omega)}^{2} \\
& \quad \leq c_{1}\left(\|f\|_{L^{2}\left(I, L_{x}^{2}(\Omega)\right)}^{2}+\left\|u_{0}\right\|_{V_{\sigma}^{1}(\Omega)}^{2}+\left\|u_{1}\right\|_{L_{x}^{2}(\Omega)}^{2}\right),
\end{aligned}
$$

where

$$
c_{1}=(1+2 b) \exp \left(\max \left(10 T^{2}+5 T+4,1+b\right) T\right) .
$$

Since the right-hand side of the obtained inequality is independent of $\tau$, we take the supremum on the left-hand side with respect to $\tau$ from 0 to $T$. Hence, estimate (3.1) holds with $c=c_{1}^{1 / 2}$.

Since we have no information concerning $R(L)$ except that $R(L) \subset F$, we must extend $L$ so that estimate (3.1) holds for the extension and its range is the whole space. To this end, we establish the following proposition. 
Proposition 3.2. The operator L from B into $F$ has a closure.

Proof. The proof is analogous to that in [8, Proposition 1].

We denote by $\bar{L}$ the closure of $L$.

Definition 3.3. A solution of the equation

$$
\bar{L} u=\left(f, u_{0}, u_{1}\right)
$$

is called a strongly generalized solution of problem (2.3).

Since points of the graph of $\bar{L}$ are limits of sequences of points of the graph of $L$, we extend (3.1) by taking the limits as follows.

Corollary 3.4. For any function $u \in D(\bar{L})$, the following estimate holds:

$$
\|u\|_{B} \leq c\|\bar{L} u\|_{F^{\prime}}
$$

where $c$ is the constant in Theorem 3.1.

Corollary 3.4 asserts that the operator $\bar{L}$ is injective and that the linear operator $\bar{L}^{-1}$ is continuous from the range $R(\bar{L})$ of $\bar{L}$ onto $B$, from which we have the following corollary.

Corollary 3.5. If a strongly generalized solution exists, it is unique and depends continuously on $\mathcal{F}=\left(f, u_{0}, u_{1}\right)$.

Corollary 3.6. The range $R(\bar{L})$ of the operator $\bar{L}$ is closed in $F$ and equals to the closure $\overline{R(L)}$ of $R(L)$, that is, $R(\bar{L})=\overline{R(L)}$.

\section{Existence of the solution}

Now, we are in a position to state the main result.

THEOREM 4.1. Problem (2.3) possesses a unique strongly generalized solution verifying

$$
\begin{gathered}
u \in C\left(\bar{I}, V_{\sigma}^{1}(\Omega)\right), \\
\frac{\partial u}{\partial t} \in C\left(\bar{I}, L_{x}^{2}(\Omega)\right) .
\end{gathered}
$$


Moreover, $u$ and $\partial u / \partial t$ depend continuously on the right-hand side of (2.3a) and on the initial conditions (2.3b) and (2.3c), that is,

$$
\begin{aligned}
\|u\|_{C\left(\bar{I}, V_{\sigma}^{1}(\Omega)\right)} & \leq c\left(\|f\|_{L^{2}\left(I, L_{x}^{2}(\Omega)\right)}+\left\|u_{0}\right\|_{V_{\sigma}^{1}(\Omega)}+\left\|u_{1}\right\|_{L_{x}^{2}(\Omega)}\right), \\
\left\|\frac{\partial u}{\partial t}\right\|_{C\left(\bar{I}, L_{x}^{2}(\Omega)\right)} & \leq c\left(\|f\|_{L^{2}\left(I, L_{x}^{2}(\Omega)\right)}+\left\|u_{0}\right\|_{V_{\sigma}^{1}(\Omega)}+\left\|u_{1}\right\|_{L_{x}^{2}(\Omega)}\right) .
\end{aligned}
$$

Proof. According to Corollary 3.6, we deduce that to prove the existence of the strongly generalized solution, it suffices to show that $R(L)$ is everywhere dense in $F$; in other words, $\bar{L}$ is injective. To this end, we firstly establish the density in a special case.

Proposition 4.2. If

$$
(\mathcal{L} u, \omega)_{L^{2}\left(I, L_{x}^{2}(\Omega)\right)}=0,
$$

for all $u \in D_{0}(L)=\left\{u / u \in D(L): \ell_{i} u=0(i=0,1)\right\}$ and for some $\omega \in L^{2}(I$, $\left.L_{x}^{2}(\Omega)\right)$, then $\omega$ vanishes almost everywhere in $Q$.

Proof. From (4.3), we have

$$
\left(\frac{\partial^{2} u}{\partial t^{2}}, \omega\right)_{L^{2}\left(I, L_{x}^{2}(\Omega)\right)}=\left(\frac{\partial}{\partial x}\left(x \frac{\partial u}{\partial x}\right), \omega\right)_{L^{2}\left(I, L^{2}(\Omega)\right)} .
$$

As equality (4.3) holds for any function $u \in D_{0}(L)$, we can express it in a special form. First, we set

$$
u=\Im_{t}((t-\tau) z)
$$

where $z, x(\partial z / \partial x),(\partial / \partial x)(x(\partial z / \partial x)),(\partial / \partial x)\left(x\left(\partial \Im_{t} z / \partial x\right)\right) \in L^{2}\left(I, L^{2}(\Omega)\right)$; further $z$ satisfies $(2.3 \mathrm{~d}),(2.3 \mathrm{e})$, and the following condition:

$$
z \in\left[\frac{x-b}{T^{2}} e^{t / T}, \frac{x-b}{T^{2}} e^{-t / T}\right]
$$

Thus, we obtain

$$
\begin{aligned}
(z, \omega)_{L^{2}\left(I, L_{x}^{2}(\Omega)\right)} & =\left(\frac{\partial}{\partial x}\left(x \frac{\partial \Im_{t}((t-\tau) z)}{\partial x}\right), \omega\right)_{L^{2}\left(I, L^{2}(\Omega)\right)} \\
& =\left(\frac{\partial}{\partial x}\left(x \frac{\partial z}{\partial x}\right), \Im_{t}^{*}((\tau-t) \omega)\right)_{L^{2}\left(I, L^{2}(\Omega)\right)}
\end{aligned}
$$


Since the left-hand side of (4.7) is a continuous linear functional of $z$, hence $\Im_{t}^{*} \omega, \Im_{t}^{*}((\tau-t) \omega) \in L^{2}\left(I, L^{2}(\Omega)\right)$ such that

$$
\frac{\partial \Im_{t}^{*}((\tau-t) \omega)}{\partial x}, \quad \frac{\partial}{\partial x}\left(x \frac{\partial \Im_{t}^{*}((\tau-t) \omega)}{\partial x}\right) \in L^{2}(Q),\left.\quad \Im_{t}^{*}((\tau-t) \omega)\right|_{x=b}=0
$$

From above, we introduce the function

$$
\omega=(T-t)^{2} z
$$

and replace it in (4.7); we get

$$
\int_{Q} x(T-t)^{2} z^{2} d x d t=\int_{Q}(T-t)^{2} \frac{\partial}{\partial x}\left(x \frac{\partial \Im_{t}((t-\tau) z)}{\partial x}\right) z d x d t
$$

Integrating by parts the right-hand side of (4.10), we obtain

$$
\begin{aligned}
\int_{Q}(T- & t)^{2} \frac{\partial}{\partial x}\left(x \frac{\partial \Im_{t}((t-\tau) z)}{\partial x}\right) z d x d t \\
= & \left.\int_{0}^{T}(T-t)^{2} x \frac{\partial \Im_{t}((t-\tau) z)}{\partial x} z\right|_{0} ^{b} d t \\
& -\int_{Q}(T-t)^{2} x \frac{\partial \Im_{t}((t-\tau) z)}{\partial x} \frac{\partial z}{\partial x} d x d t \\
= & -\left.\int_{\Omega}(T-t)^{2} x \frac{\partial \Im_{t}((t-\tau) z)}{\partial x} \frac{\partial \Im_{t} z}{\partial x}\right|_{0} ^{T} d x \\
& +\int_{Q}(T-t)^{2} x\left(\frac{\partial \Im_{t} z}{\partial x}\right)^{2} d x d t \\
& -2 \int_{Q}(T-t) x \frac{\partial \Im_{t}((t-\tau) z)}{\partial x} \frac{\partial \Im_{t} z}{\partial x} d x d t \\
= & \int_{Q}(T-t)^{2} x\left(\frac{\partial \Im_{t} z}{\partial x}\right)^{2} d x d t \\
& -\left.\int_{\Omega}(T-t) x\left(\frac{\partial \Im_{t}((t-\tau) z)}{\partial x}\right)^{2}\right|_{0} ^{T} d x \\
= & \int_{Q}(T-t)^{2} x\left(\frac{\partial \Im_{t} z}{\partial x}\right)^{2} d x d t-\int_{Q} x\left(\frac{\partial \Im_{t}((t-\tau) z)}{\partial x}\right)^{2} d x d t \\
& \left.-\int_{Q} x(t-\tau) z\right) \\
& \\
& \\
&
\end{aligned}
$$


Substituting (4.11) into (4.10) and estimating from above the right-hand side, we obtain

$$
\int_{Q} x(T-t)^{2} z^{2} d x d t \leq T^{2}\left\|\frac{\partial z}{\partial x}\right\|_{B_{2}^{1}\left(I, L_{x}^{2}(\Omega)\right)}^{2}-\left\|\frac{\partial z}{\partial x}\right\|_{B_{2}^{2}\left(I, L_{x}^{2}(\Omega)\right)}^{2} .
$$

Thanks to condition (4.6), we deduce that the right-hand side is less than zero. Consequently, we have

$$
\int_{Q} x(T-t)^{2} z^{2} d x d t \leq 0
$$

from which we conclude that $\omega$ vanishes almost everywhere in $Q$.

Now consider the general case. Since $F$ is a Hilbert space, the density of $R(L)$ in $F$ is equivalent to the property that orthogonality of a vector $W=\left(\omega, \omega_{0}, \omega_{1}\right) \in F$ to the range $R(L)$, namely, the integral identity

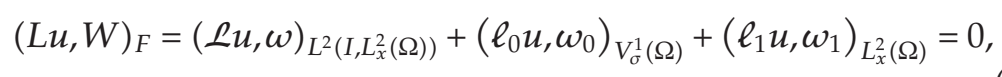

implies $W \equiv 0$. Putting $u \in D_{0}(L)$ in (4.14), we obtain

$$
(\mathcal{L} u, \omega)_{L^{2}\left(I, L_{x}^{2}(\Omega)\right)}=0, \quad u \in D_{0}(L) .
$$

Hence, Proposition 4.2 implies that $\omega \equiv 0$. Thus, (4.14) takes the form

$$
\left(\ell_{0} u, \omega_{0}\right)_{V_{\sigma}^{1}(\Omega)}+\left(\ell_{1} u, \omega_{1}\right)_{L_{x}^{2}(\Omega)}=0 .
$$

As $\ell_{0}, \ell_{1}$ are independent and the sets $R\left(\ell_{0}\right), R\left(\ell_{1}\right)$ are everywhere dense in the spaces $V_{\sigma}^{1}(\Omega)$ and $L_{x}^{2}(\Omega)$, respectively, then relation (4.16) implies that $\omega_{0} \equiv 0$ and $\omega_{1} \equiv 0$. Hence, $W \equiv 0$, and thus $\overline{R(L)}=F$.

\section{Conclusion}

In this paper, we proved the existence and uniqueness of a strongly generalized solution, in weighted spaces, of problem (2.3) in the sense of Definition 3.3. The weight here appears, on the one hand, because of a singular coefficient of the equation, and on the other hand, comes to place for the annihilation of inconvenient terms during integration by parts. Besides, $u$ and $\partial u / \partial t$ depend continuously upon the right-hand side of (2.3a) and on the initial conditions (2.3b) and (2.3c). Note that the strongly generalized solution is also a weak solution [10]. 
The used method is one of the most efficient functional analysis methods for solving linear PDE with nonlocal boundary conditions, the socalled energy-integral method or a priori estimates method. That is due to the fact that we construct for each problem suitable multiplicators, which provides the a priori estimate, from which it is possible to establish the solvability of the problem. However, the great flexibility of the method has its own disadvantage: the major difficulty of the choice of the adequate multiplicators to the considered problems, which is the crucial step of the establishment of the a priori estimate.

\section{Acknowledgments}

The author wishes to express his gratitude to the referee for his valuable suggestions which led to the improvement of the paper. This work was carried out at The Abdus Salam International Centre for Theoretical Physics, Trieste, Italy.

\section{References}

[1] S. A. Beilin, Existence of solutions for one-dimensional wave equations with nonlocal conditions, Electron. J. Differential Equations 2001 (2001), no. 76, 1-8.

[2] A. Bouziani, Mixed problem with boundary integral conditions for a certain parabolic equation, J. Appl. Math. Stochastic Anal. 9 (1996), no. 3, 323-330.

[3]__ Mixed problem with integral condition for certain partial differential equations, Ph.D. thesis, Constantine University, Constantine, 1996 (French).

[4] Solution forte d'un problème mixte avec une condition non locale pour une classe d'équations hyperboliques [Strong solution of a mixed problem with a nonlocal condition for a class of hyperbolic equations], Acad. Roy. Belg. Bull. Cl. Sci. (6) 8 (1997), no. 1-6, 53-70 (French).

[5] On the solvability of nonlocal pluriparabolic problems, Electron. J. Differential Equations 2001 (2001), no. 21, 1-16.

[6] - Initial-boundary value problem with a nonlocal condition for a viscosity equation, Int. J. Math. Math. Sci. 30 (2002), no. 6, 327-338.

[7] On a class of nonclassical hyperbolic equations with nonlocal conditions, J. Appl. Math. Stochastic Anal. 15 (2002), no. 2, 135-153.

[8] On the solvability of parabolic and hyperbolic problems with a boundary integral condition, Int. J. Math. Math. Sci. 31 (2002), no. 4, 201-213.

[9] R. Dautray and J.-L. Lions, Analyse Mathématique et Calcul Numérique pour les Sciences et les Techniques. Vol. 8 [Mathematical Analysis and Computing for Science and Technology], INSTN: Collection Enseignement, Masson, Paris, 1988 (French).

[10] A. A. Dezin, Partial Differential Equations, Springer Series in Soviet Mathematics, Springer-Verlag, Berlin, 1987.

[11] L. Gårding, Cauchy's Problem for Hyperbolic Equations, University of Chicago Lecture Notes, University of Chicago, Illinois, 1957. 


\section{On hyperbolic equation with an integral condition}

[12] S. Mesloub and A. Bouziani, On a class of singular hyperbolic equation with a weighted integral condition, Int. J. Math. Math. Sci. 22 (1999), no. 3, 511519.

[13] - Mixed problem with integral conditions for a certain class of hyperbolic equations, J. Appl. Math. 1 (2001), no. 3, 107-116.

[14] A. M. Nakhushev, An approximate method for solving boundary value problems for differential equations and its application to the dynamics of ground moisture and ground water, Differentsial'nye Uravneniya 18 (1982), no. 1, 72-81 (Russian).

[15] L. S. Pulkina, A non-local problem with integral conditions for hyperbolic equations, Electron. J. Differential Equations 1999 (1999), no. 45, 1-6.

[16] - On the solvability in $L_{2}$ of a nonlocal problem with integral conditions for a hyperbolic equation, Differential Equations 36 (2000), no. 2, 316-318 (Russian).

Abdelfatah Bouziani: Department of Mathematics, The Larbi Ben M'hidi University Centre, P.O. Box. 565, Oum El Bouagui 04000, Algeria

E-mail address: af_bouziani@hotmail.com 


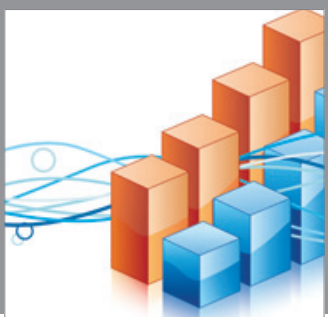

Advances in

Operations Research

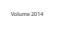

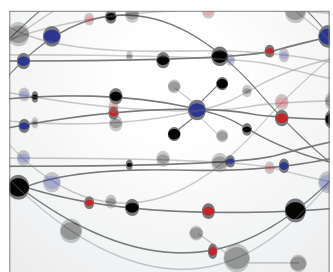

\section{The Scientific} World Journal
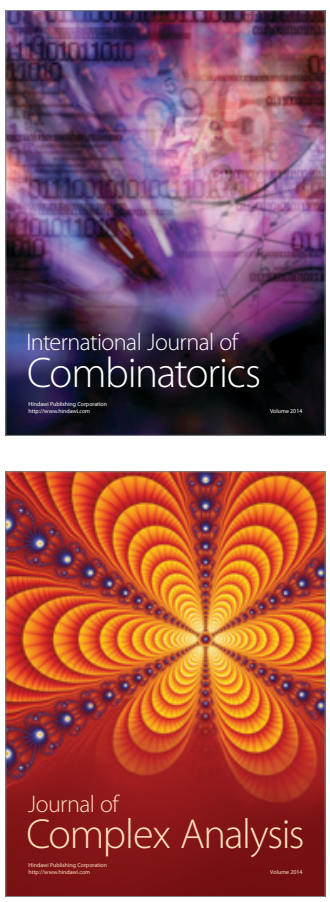

International Journal of

Mathematics and

Mathematical

Sciences
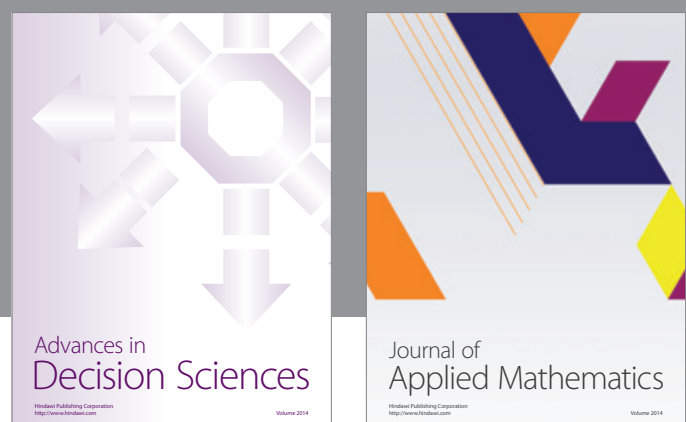

Journal of

Applied Mathematics
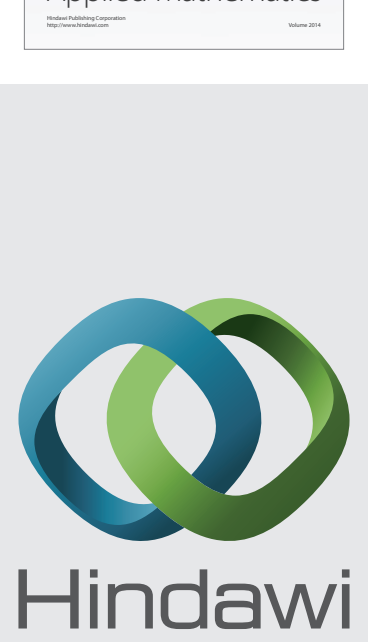

Submit your manuscripts at http://www.hindawi.com
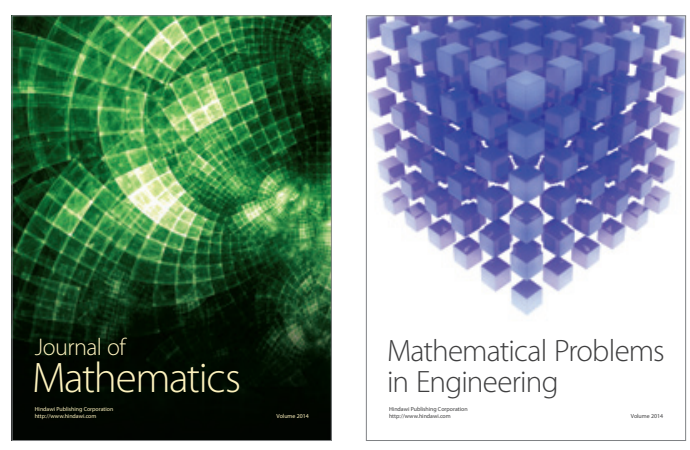

Mathematical Problems in Engineering
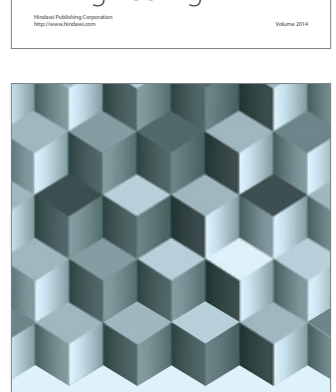

Journal of

Function Spaces
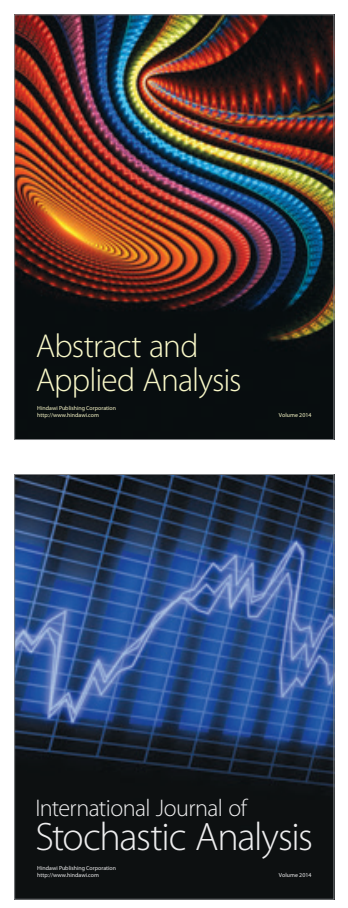

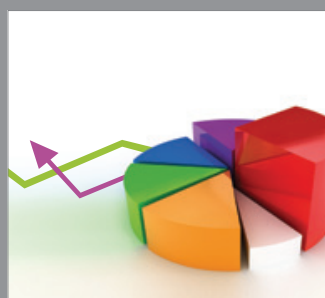

ournal of

Probability and Statistics

Promensencen
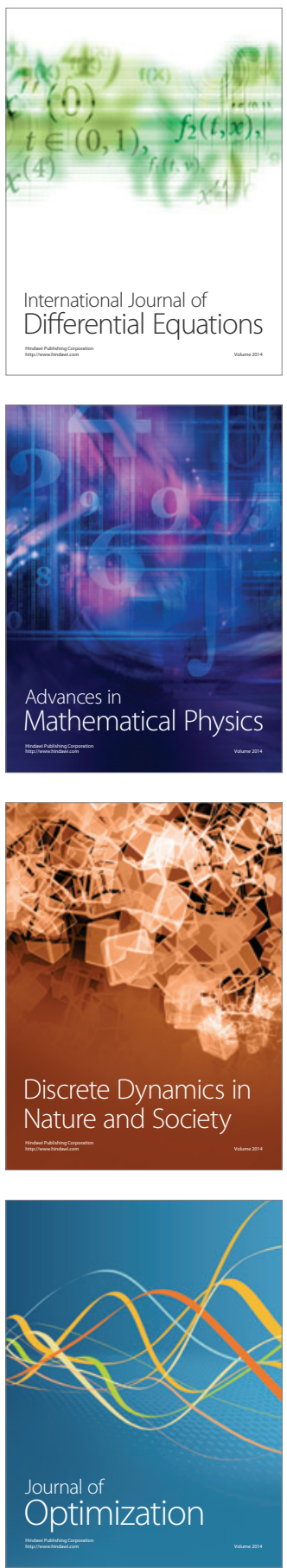